\title{
Searches for diffuse fluxes of cosmic neutrinos with the ANTARES telescope
}

\author{
Luigi Antonio Fusco ${ }^{1,2, a}$ on behalf of the ANTARES Collaboration \\ ${ }^{1}$ Dipartimento di Fisica e Astronomia dell'Università, Viale Berti-Pichat 6/2, 40127 Bologna, Italy \\ ${ }^{2}$ INFN - Sezione di Bologna, Viale Berti-Pichat 6/2, 40127 Bologna, Italy
}

\begin{abstract}
In this proceedings we report on the status of searches for diffuse fluxes of cosmic neutrinos with the ANTARES neutrino telescope data. A complete overview of full sky searches will be given, together with the analysis of possible diffuse neutrino emission from regions such as the Fermi Bubbles or the Galactic Plane.
\end{abstract}

\section{Introduction}

Neutrinos are predicted to be produced nearby the expected Cosmic Rays (CR) accelerators, such as Supernova Remnants, Active Galactic Nuclei or Gamma Ray Bursts. A diffuse flux of cosmic neutrinos is expected from unresolved individual sources. The energy spectrum of these neutrinos should be similar to that of primary CRs, produced by Fermi shock acceleration, and flatter than the observed atmospheric neutrino background.

The IceCube Collaboration has reported [1] the observation of an flavour diffuse and isotropic excess of high energy neutrinos, not compatible with atmospheric expectations. This observation has opened the path to high energy neutrino astronomy.

The ANTARES detector [2] is currently the largest neutrino telescope in the Northern Hemisphere, located at a depth of $2475 \mathrm{~m}$ in the Mediterranean Sea, $40 \mathrm{~km}$ from Toulon, France and continuously operated since 2007. It consists of almost 900 photomultiplier tubes (PMT), distributed on 12 vertical strings anchored to the sea bed. It detects neutrinos using the Cherenkov light emitted by particles produced after neutrino interactions in the surroundings of the detector. Charged current (CC) $v_{\mu}$ interactions, where a muon straight track is produced, can be properly reconstructed in direction with a good pointing accuracy [3]. All flavours neutral current (NC) interactions, as well as CC $v_{e}$ and $v_{\tau}$ interactions can be reconstructed by dedicated shower algorithms.

The main background in searches for diffuse fluxes of a cosmic signal is given by atmospheric neutrinos and muon bundles produced in CR air showers. To reduce the background from down-going muons only tracks reconstructed as upward-goinh are selected, as only neutrinos can traverse the Earth. Nonetheless many wrongly reconstructed atmospheric muons can survive this directional selection, but a proper cut on the reconstruction quality parameter can help to reduce this background.

ae-mail: lfusco@bo.infn.it

(C) The Authors, published by EDP Sciences. This is an Open Access article distributed under the terms of the Creative Commons Attribution License 4.0 (http://creativecommons.org/licenses/by/4.0/). 


\section{Full sky searches}

In this section full sky searches of cosmic neutrinos will be presented, both in the CC $v_{\mu}$ channel (Sect. 2.1) and for all flavours neutrino interactions reconstructed as shower events (Sect. 2.2). Along with this, an estimation of the atmospheric $v_{\mu}$ energy spectrum - the neutrino background for cosmic searches - will be given in Sect. 2.3. Blinded analysis procedures have been followed, with only $10 \%$ of data available for tests and data/Monte Carlo comparison. The good agreement between data and Monte Carlo allowed for a final cut choice based on simulations.

\subsection{Muon neutrinos diffuse flux analysis}

An update of the analysis on diffuse $v_{\mu}$ fluxes reported in [4], with two additional years of ANTARES data (2007-2011), is presented. The equivalent livetime is 885 days, about a factor three larger than the previous analysis.

Most of the down-going atmospheric muon events are rejected applying a preliminary cuts on the reconstructed zenith angle $\left(\theta_{z e n}>90^{\circ}\right)$ and on the angular error estimation $\beta\left(\beta<0.5^{\circ}\right)$. To further reduce the atmospheric background combined cut on reconstruction quality parameter, $\Lambda$, and on the number of hit related to the reconstructed track, $\mathrm{N}_{h i t}$, has been applied thus reducing the muon background level well below $1 \%$.

Atmospheric neutrinos can be rejected by applying an energy related cut, based on the estimation of the muon energy loss in the detector [5]. The optimal cut on this variable is chosen through a Model Rejection Factor (MRF) procedure [6], determining the best sensitivity flux $\mathrm{E}^{2} \hat{\Phi}_{\text {track }}^{90 \%}=4.7 \cdot 10^{-8} \mathrm{GeV} \mathrm{cm}^{-2} \mathrm{~s}^{-1} \mathrm{sr}^{-1}$. After the final cut 8.4 events are expected from the background and 1.4 signal events should be observed from the signal reported in [1]. The $90 \%$ of the expected signal after cuts has energy in the range from $45 \mathrm{TeV}$ to $10 \mathrm{PeV}$.

After unblinding 8 events are found in data. Using the method from [7] the upper limit at $90 \%$ confidence level is $\mathrm{E}^{2} \Phi_{\text {track }}^{90 \%}=5.1 \cdot 10^{-8} \mathrm{GeV} \mathrm{cm}^{-2} \mathrm{~s}^{-1} \mathrm{sr}^{-1}$ (Fig. 1). The systematic error on the background is taken from the observed normalization of atmospheric neutrino flux measurement shown in section Sect. 2.3 with respect to the Bartol flux [8] model adopted in this work. Systematics on the signal are taken into account by varying water properties, PMT efficiency and angular acceptance in the simulation.

\subsection{All flavour diffuse flux analysis with showers}

All events reconstructed with the shower algorithm of [9] are considered in this analysis. A vertex likelihood fit, followed by an energy and direction fit are performed by the reconstruction method. This leads to an energy resolution of $0.2-0.3$ for the $\log _{10} \mathrm{E}_{\text {shower }}$ and a median angular resolution of $6^{\circ}$ in the hundred $\mathrm{TeV}$ region.

The analysis collects all data from 2007 to 2012, with a total livetime of 1247 days. An event pre-selection is done using a cut on the vertex likelihood and requiring signal hits to be present on at least three detector lines. This selection significantly reduces the the contribution from track events, including atmospheric muon background. Additionally the reconstructed vertex position is required not to be closer than $15 \mathrm{~m}$ to any PMT, to exclude spurious events from sparks in optical modules.

The MRF optimisation is done on the fitted shower energy and zenith angle. The optimal cut is found to be $\mathrm{E}_{\text {shower }}>10 \mathrm{TeV}$ and $\theta>94^{\circ}$, leading to a sensitivity with $\mathrm{E}^{2} \hat{\Phi}_{\text {showers }}^{90 \%}=$ $2.2_{-0.7}^{+0.9} \cdot 10^{-8} \mathrm{GeV} \mathrm{cm}^{-2} \mathrm{~s}^{-1} \mathrm{sr}^{-1} .5 \pm 3$ background events are expected, and the IceCube flux would correspond to $2.1_{-0.7}^{+0.5}$ cosmic events added to the atmospheric expectations. 8 events 


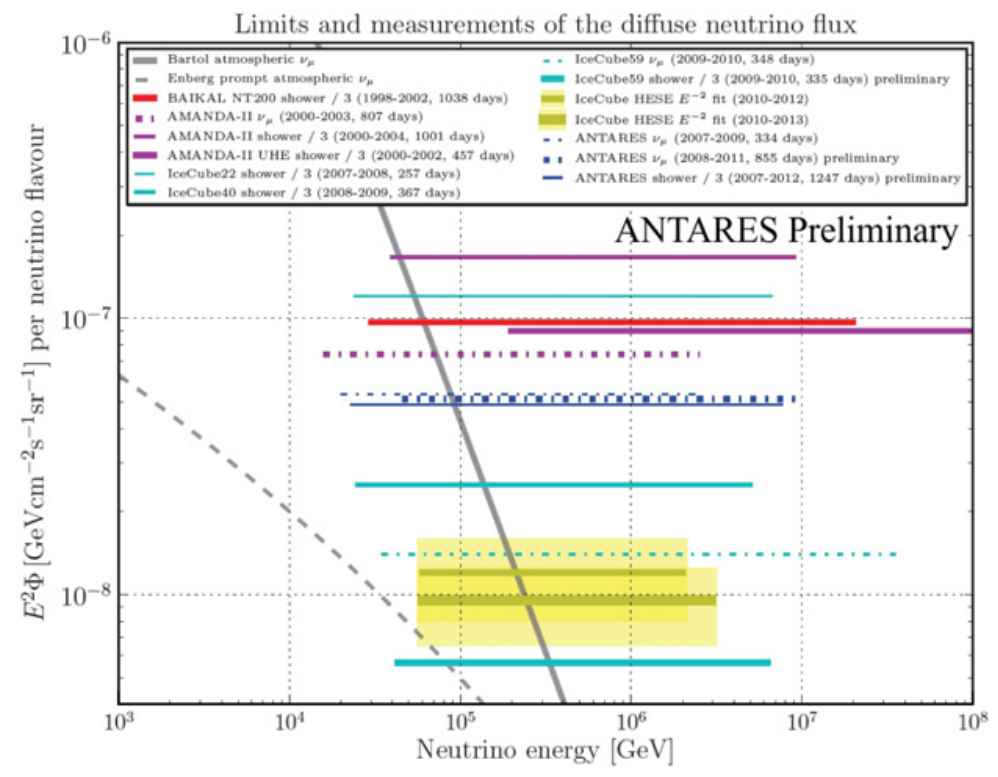

Figure 1. Summary of diffuse flux limits from various experiments in the different detection channels for neutrinos, as reported in the legend.

are observed in data after unblinding. The excess over background has a significance of $1.5 \sigma$. Considering an $\mathrm{E}^{-2}$ spectrum, the $90 \%$ C.L. upper limit on the cosmic neutrino diffuse flux is $\mathrm{E}^{2} \Phi_{\text {showers }}^{90 \%}=4.9 \cdot 10^{-8} \mathrm{GeV} \mathrm{cm}^{-2} \mathrm{~s}^{-1} \mathrm{sr}^{-1}$, including systematics on signal and background. The validity energy range for this limit is $23 \mathrm{TeV}-7.8 \mathrm{PeV}$ (Fig. 1).

\subsection{Atmospheric neutrino energy spectrum}

Atmospheric neutrinos are sources of background in neutrino telescopes, but their observation can give valuable information on the detector performances as well as on the systematics affecting the energy measurement. Moreover great uncertainties are present at high energies, both in conventional fluxes - from charged $\pi$ and K meson decays - and in prompt fluxes from decaying charmed particles. Measuring their flux can thus improve the knowledge of the neutrino detection itself.

Using the same data sample of Sect. 2.1 two different analysis have been performed, with different selection cuts and unfolding methods and with a complete evaluation of systematic errors. Analogous results in the evaluation of the atmospheric neutrino spectrum from $100 \mathrm{GeV}$ to $200 \mathrm{TeV}$ [5] are achieved in both analyses. The observed spectrum (Fig. 2) is compatible with the expectations from conventional fluxes, being $25 \%$ higher in the overall normalization than the flux of [8] but within reported uncertainties. No limit on the prompt contribution can be given as large uncertainties are present at the highest energies.

\section{Special regions}

In this section searches devoted to possible extended neutrino emission regions will be shown. In particular the so called Fermi Bubbles and the Galactic Plane regions have been analysed, as ANTARES has an excellent coverage of these areas of the sky. For these analyses an on/off-zone procedure has been followed. Considering regions of the sky with the same 

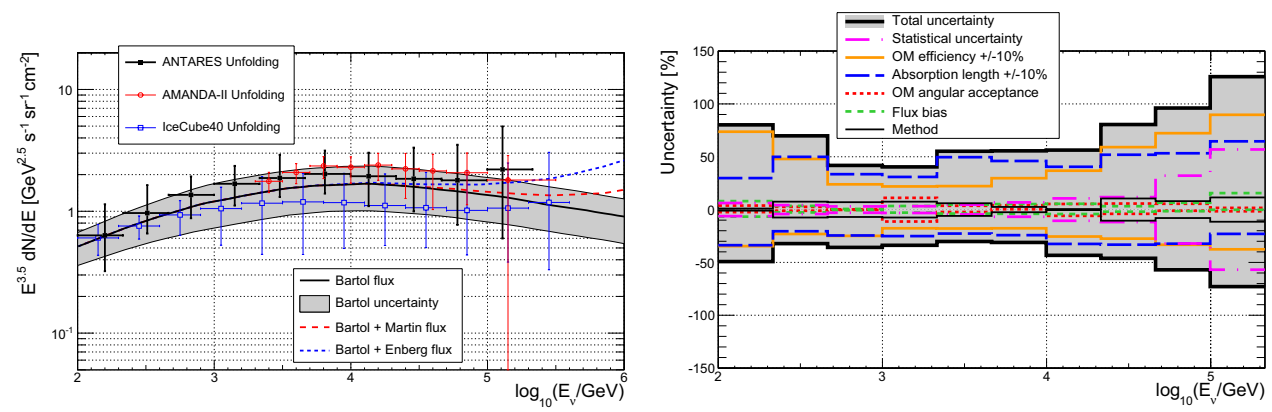

Figure 2. Left: measurement of the atmospheric muon neutrino energy spectrum compared with theoretical predictions (solid line for conventional, dashed for prompt) and experimental results from AMANDA and IceCube. Right: uncertainties on the atmospheric neutrino energy spectrum measurement, considering different effects on water and OM properties, as well as the uncertainties on the underlying spectrum and deriving from the unfolding methods. Color code in the legend.

shape, detector efficiency and visibility of the expected signal regions the background has been estimated and then compared to the actual observation in the signal region. In absence of a signal, the observed number of events in on/off-zones should be statistically compatible.

\subsection{Fermi bubbles}

Fermi telescope data $[10,11]$ have revealed the presence of two large $\gamma$-ray emission regions above and below the Galactic plane. If hadronic mechanisms are responsible for the production of such a signal, diffuse neutrino emissions are expected from these regions with various possible energy cut-offs, from few to some hundreds of TeV [12].

Data collected in the $v_{\mu}$ CC channel with the ANTARES telescope from 2007 to 2011 are considered in the analysis [14]. The event selection is based on cuts on the quality of upgoing reconstructed tracks and on the energy estimation through an Artificial Neural Network (ANN) [13]. The final selection cut, obtained minimising the MRF, is $\Lambda>-5.14$ and $\mathrm{E}_{A N N}>$ $11 \mathrm{TeV}$, when optimizing for a neutrino flux having a cut-off at $100 \mathrm{TeV}$. After the unblinding of the on-zone, 16 events are observed, while 11 are expected, on average, from the off-zones. The significance of this excess can be estimated, following the prescription of [15], as $1.2 \sigma$ and upper limits are calculated (Fig. 3).

\subsection{Galactic plane}

A diffuse neutrino flux is expected from the decays of charged mesons produced in CR interactions in the interstellar medium in the Galactic Plane. The corresponding emission from neutral mesons is clearly visible in $\gamma$-ray observation of the sky [16]. Different models for the CR propagation are proposed (e.g. [17-19]) as well as direct computations from Fermi data [16], each leading to a different neutrino flux expectation but in agreement within a factor $\sim 2$. NoDrift models $[17,18]$ do not take into account magnetic fields while the Drift model [19], doing so, produces an enhancement of the neutrino flux in the Galactic Centre region.

Muon neutrinos collected by ANTARES from 2007 to 2011 have been analysed [20] following the MRF procedure to set an optimal cut on the area of the on-zone $\left(78^{\circ}\right.$ size in longitude and $9^{\circ}$ size in latitude centered at the Galactic Centre) and on the quality and energy of reconstructed tracks $\left(\Lambda>-5.6, \beta<8^{\circ}\right.$ and $\left.\mathrm{E}_{A N N}>250 \mathrm{GeV}\right)$. The 8 selected 

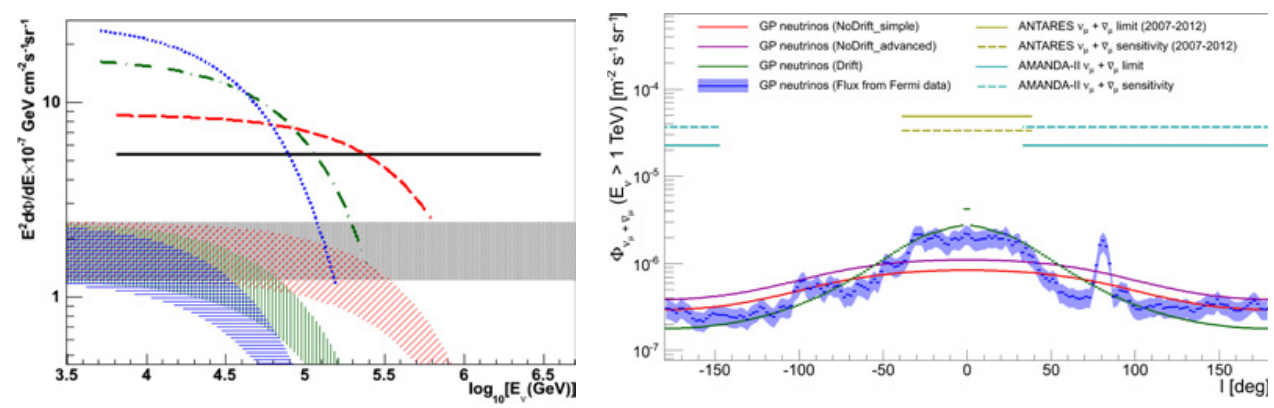

Figure 3. Left: upper limit calculation for neutrino fluxes from the Fermi Bubbles for different energy cut-offs $(\infty, 100 \mathrm{TeV}, 50 \mathrm{TeV}, 10 \mathrm{TeV}$ - black, red, green and blue lines). Shaded areas are for theoretical predictions with the various cuf-offs. Right: upper limits and sensitivity for Galactic Plane analyses from ANTARES and AMANDA, compared to theoretical models as reported in the legend - see text for explanations.

off-zones show on average 166 events, and 177 are observed in the on-zone after unblinding. The significance of this excess is $0.8 \sigma$, and the calculated upper limits are reported in Fig. 3.

\section{Conclusions}

The ANTARES neutrino telescope is in its $7^{\text {th }}$ year of operation. Despite its moderate size, but thanks to its location and good event reconstruction performances, it yields good diffuse flux sensitivity in the relevant range and the best limits for the Galactic Plane and the Fermi bubble regions. In the near future more data will be added and joint track and shower analyses will be performed. The next generation KM3NeT neutrino telescope will further improve the results for diffuse flux sensitivities.

\section{References}

[1] M. G. Aartsen et al., Phys. Rev. Lett. 113, 101101 (2013)

[2] M. Ageron et al., Nucl. Instrum. Methods A 656, 11 (2011)

[3] S. Adrián-Martínez et al. Astrophys. J 760, 53 (2012)

[4] J.A. Aguilar et al., Phys. Lett. B 696, 16 (2011)

[5] S. Adrián-Martínez et al. Eur. Phys. J. C 73, 2606 (2013)

[6] G.C. Hill and K. Rawlins, Astropart. Phys. 19, 393 (2003)

[7] J. Conrad et al., Phys. Rev D 67, 012002 (2003)

[8] G.D. Barr et al, Phys. Rev. D 74, 094009 (2006)

[9] F. Folger, PhD thesis, FAU (2014)

[10] M. Su et al., Astrophys. J. 724, 1044 (2010)

[11] M. Ackermann et al., Astrophys. J. 793, 64 (2014)

[12] R. M. Crocker and F. Aharonian, Phys. Rev. Lett. 106, 101102 (2011)

[13] J. Schnabel, Nucl. Instrum. Methods A 725, 106 (2013)

[14] S. Adrián-Martínez et al. Eur. Phys. J. C 74, 2701 (2014)

[15] T. P. Li and Y. Q. Ma, Astrophys. J. 272, 317 (1983)

[16] M. Ackermann et al., Astrophys. J. 750, 3 (2012)

[17] G. Ingelman and M. Thunman, Arxiv preprint hep-ph/9604286 (1996)

[18] J. Candia and E. Roulet, JCAP 09, 005 (2003)

[19] J. Candia, JCAP 11, 002 (2005)

[20] E. Visser, $\mathrm{PhD}$ thesis, University of Leiden, to be published 